\title{
ONOMÁVAIN
}

Revista de lingüística, filología y traducción

\section{Hacia un glosario de hispanismos en un corpus de novela rosa inglesa contemporánea ${ }^{1}$}

Towards a glossary of Hispanicisms

in a corpus of contemporary

English romance novels

\section{María Isabel González Cruz \\ Universidad de las Palmas de Gran Canaria \\ España}

\section{(C) $($ ii) $\ominus$}

María Isabel González Cruz: Departamento de Filología Moderna, Universidad de las Palmas de Gran Canaria, España. | E-mail: isabel.gonzalezcruz@ulpgc.es 


\section{Resumen}

Este artículo pretende justificar la creación de un glosario comentado con los hispanismos que aparecen en un corpus de novela rosa inglesa compilado para el Proyecto de Investigación FFl2014-53962-P. Al estar total o parcialmente ambientadas en una zona hispanohablante, las Islas Canarias, las obras de este corpus muestran una tendencia notable a la inserción de palabras, expresiones e incluso frases en español. Además de describir las pautas para la elaboración de este inventario léxico, en este trabajo preliminar ofrecemos un avance de los registros que incluirá el futuro glosario, con los hispanismos detectados en 30 novelas publicadas entre 1955 y 2004. Con esta aportación pretendemos contribuir a los estudios del papel del español como lengua emisora de préstamos.

Palabras clave: hispanismos; préstamos; glosario; novela rosa; bibliografía inglesa sobre Canarias.

\section{Abstract}

This paper aims to justify the building of a glossary or annotated inventory of all the Hispanicisms used in a corpus of English popular romance fiction novels compiled for research project FFI2014-53962-P. An examination of these texts, which are totally or partially set in a Spanish-speaking area, the Canary Islands, proves their tendency to include Spanish words, phrases and even sentences. In this preliminary work, we will describe the guidelines we are using for the compilation and classification of the Hispanicisms found in 30 novels, published between 1955 and 2004, and will also list the entries to be included in the glossary. The work is conceived as a contribution to the studies on the role of Spanish as a loan-giver language.

Keywords: Hispanicisms; borrowing; glossary; popular romance fiction novels; English bibliography on the Canaries.

1 El presente artículo surge del proyecto interdisciplinar FFI2014-53962-P, Discursos, género e identidad en un corpus de novela rosa inglesa ambientada en Canarias y otras islas atlánticas. Agradezco aquí la financiación recibida del MINECO (Ministerio de Economía y Competitividad del Gobierno de España) para su realización. 


\section{Introducción}

El estudio de la influencia del español en la lengua inglesa cuenta ya con una larga tradición (Peñuelas, 1964; Lope-Blanch, 1990; Rodríguez González, 1995, 1996; Algeo, 1996; Cannon, 1996; Gooch, 1996; Lodares, 1996; Alvar, 2000; Roca, 2000, entre otros). El interés por este asunto, lejos de frenarse con la imparable hegemonía del inglés como lengua fuente de préstamos a nivel mundial, parece haberse incrementado en los últimos años, con nuevas investigaciones que no solo abordan cuestiones como el papel del español en la formación del léxico inglés (Rodríguez González, 2001; Lacorte y Leeman, 2009; González Cruz, 2011; Schultz, 2018), sino otros temas de permanente interés, como pueden ser los patrones que determinan el intercambio léxico entre ambas lenguas (Muñoz-Basols y Salazar, 2016), las actitudes de los hablantes hacia la incorporación de ese léxico (Anderson y Toribio, 2007; Guijarro y Ruiz, 2004), cómo afectan las políticas lingüísticas y la globalización (Mar-Molinero, 2000, 2004; Ferreira Martins, 2016), o bien la identidad bilingüe y multilingüe de los hispano-hablantes que se encuentran en situaciones de contacto, sobre todo con el inglés de los Estados Unidos (Morales, 2002; Fairclough, 2003; Stavans, 2003; Ardila, 2005; Ortiz y Lacorte, 2005; Vilar, 2008; Potowski y Rothman, 2011; Thompson y Lamboy, 2012; Dumitrescu y Pina-Rosales, 2013; Rojas y Piñón, 2014; Limerick, 2017), pero también con otras lenguas (Silva-Corvalán, 1995; Del Valle y Villa, 2006; Niño-Murcia y Rothman, 2008).

Y es que para nuestra sociedad globalizada los fenómenos del contacto de lenguas y del préstamo léxico son simples consecuencias naturales y muy cotidianas. De hecho, se sabe que con el transcurso del tiempo y el devenir histórico todas las lenguas han experimentado fenómenos como son la incorporación y la adaptación de distintas unidades léxicas tomadas de otros idiomas, así como también la desaparición de parte de su vocabulario. Hoy en día prácticamente todas las comunidades lingüísticas tienen que adaptarse a unos contextos sociales y tecnológicos fluctuantes, que traen consigo una gran cantidad de vocabulario nuevo (Picone, 1996: 10), lo que demuestra que los préstamos son el ejemplo de cambio lingüístico más consistentemente extendido (Aitchison, 1991: 16).

A pesar de su papel dominante e influyente en múltiples ámbitos, en su expansión por todo el mundo la lengua inglesa ha mostrado siempre una gran receptividad para la incorporación de palabras tomadas de las numerosas lenguas con las que ha estado en contacto (Sheard, 1954; Speake y Laflaur, 2002; Crystal, 2003; Durkin, 2014) y el español es, sin duda, una de las que ha contribuido de manera notable al enriquecimiento del vocabulario inglés (Serjeantson, 1935; Jackson y Amvela, 2004; Delahunty, 2010; Grant, 2009; Schultz, 2018). Las vías para la infiltración de hispanismos en el repertorio léxico de los hablantes anglófonos han sido muy diversas. Actualmente suelen estar ligadas al mundo de la cultura, como es el caso de la música latina y sus distintos estilos (Mar-Molinero, 2013), junto con otras manifestaciones culturales, como la lectura de textos literarios. Así, distintos investigadores han demostrado el significativo papel que para la difusión de hispanismos han tenido tanto la literatura chicana 
(Martín, 1994) como muchos relatos de viajes, por el extenso uso que de ellos han hecho en sus textos muchos autores de escritura alemana (Batista y Sarmiento, 2007), francesa (Corbella, 1991; Curell y otros, 2009) o inglesa (González Cruz y González de la Rosa, 2006; González Cruz, 2011, 2013). Otro género relevante es el de la novela rosa, un tipo de literatura popular que, aunque denostada por la crítica por su condición de popular y femenina (Sánchez-Palencia, 1997), cuenta con unos índices de venta imbatibles a nivel internacional. En estudios anteriores (González Cruz, 2015, 2017a, 2017b) hemos comprobado que, cuando están ambientadas en zonas exóticas de habla no inglesa, estas obras tienden a incluir muchos extranjerismos como parte de su técnica narrativa. Este es precisamente el caso del corpus de novelas objeto de estudio en el Proyecto de Investigación FFI2014-53962-P, en cuyo marco hemos examinado los 30 títulos que enumeramos más abajo como fuentes primarias de este estudio. Podemos confirmar aquí la tendencia en esos textos a la inserción de hispanismos, lo que sugiere que este tipo de publicaciones puede funcionar como un importante canal para la difusión de este vocabulario y la posible incorporación al repertorio léxico de su numeroso público lector a nivel internacional².

En este sentido, cabe recordar lo ya señalado por autores como Thomason (2010) o Braunmüller y House (2009: 1-9) al escribir: "lexical borrowing [...] can occur through book reading and learning by writers, teachers, students, who then pass on the new items to other people through other written texts".

En este trabajo pretendemos justificar nuestro interés por crear un glosario comentado con los hispanismos detectados en los textos del corpus que se está compilando para el mencionado proyecto. El glosario se concibe como un inventario léxico que contribuirá a los estudios del español como lengua emisora de préstamos, complementando el catálogo de trabajos ya existente sobre la incorporación de hispanismos a diversas lenguas del mundo, tales como los de Cioranescu (1987), Sabia y Achiri (2012), Giraldo-Gallego (2013), etc. El artículo se organiza como sigue: tras la introducción, explicaremos nuestros objetivos concretos, al tiempo que justificamos el interés del trabajo. La sección 3 ofrece unas breves reflexiones sobre aspectos del estudio de los préstamos. El apartado 4 describe brevemente el corpus objeto de estudio,

2 Parameswaran (1999) muestra cómo estas novelas románticas funcionan en la India como recurso para la mejora de la competencia del inglés entre sus numerosas lectoras. De hecho, una de las autoras más reconocidas del género romance, Sherry Thomas, confiesa haber aprendido inglés gracias a la lectura de novelas rosa (https://www.amazon.com/Sherry-Thomas/e/Bool/LFOGK), a partir del vocabulario de 200 palabras adquirido durante su primer año de inglés en China (http:// www.popularromaceproject.org). Igualmente, la investigadora Jayashree Kamble, de La Guardia Community College (CUNY), en los debates del International Seminar on Languages and Cultures in the Romance Novel, celebrado en la Universidad de Las Palmas de Gran Canaria en junio de 2017, confirmó que había aprendido mucho vocabulario español leyendo novelas rosa ambientadas en zonas hispanohablantes. 
la metodología y las pautas que estamos siguiendo para la elaboración del glosario. En la quinta sección aportamos, como resultado preliminar, un listado clasificado y brevemente comentado con los hispanismos registrados en los textos, y terminamos con un comentario final.

\section{Objetivos y justificación}

Los objetivos fundamentales que perseguimos en este artículo son tres:

a) Justificar el interés del glosario comentado propuesto.

b) Describir las pautas que nos están guiando en la elaboración de dicho glosario.

c) Ofrecer como adelanto el registro clasificado de los hispanismos detectados en los textos examinados.

d) Determinar qué porcentaje de los hispanismos utilizados en las novelas son préstamos ya integrados en los registros lexicográficos de la lengua inglesa, lo que de algún modo legitimaría su uso, y cuáles serían neologismos aún no incorporados.

Por otra parte, los objetivos concretos del glosario comentado son los siguientes:

a) Recopilar todos los hispanismos que se insertan en las novelas del corpus, ya sean unidades léxicas o fraseológicas, ilustrando su uso contextualizado y aportando datos lexicográficos (fecha de incorporación, categorías gramaticales, significado, etc.).

b) Comprobar la existencia de posibles equivalentes en la lengua inglesa y aportarlos, en su caso.

c) Identificar las funciones textuales que desempeñan los hispanismos y las posibles diferencias estilísticas que su uso conlleve.

d) Averiguar qué tipo de hispanismos tienden a utilizarse con mayor frecuencia en este tipo de textos, es decir, qué categorías gramaticales y qué ámbitos temáticos son más proclives a incorporarse al discurso en lengua inglesa de las novelas.

El interés de este tipo de estudios se justifica, en primer lugar, por la reconocida necesidad de llevar a cabo registros periódicos del uso del lenguaje que revelen la incorporación de nuevas voces al repertorio sociolingüístico de los hablantes (cf. Rodríguez González y Lillo Buades, 1997: 136; Turell, 1986: 39). En este sentido coincidimos con Chapman (1990: 36) cuando señala que la literatura constituye no solo un ejemplo de uso de una lengua, sino que además representa uno de los usos más frecuentes del lenguaje. Obviamente, la lengua es la materia prima de cualquier escritor y la grandeza de un autor suele medirse por la competencia que tenga en cuanto al uso del lenguaje. En palabras de Clark (1996: 3-5): "Novelists [...] rely on the written word to entertain, inform, and persuade. All these are instances of language use - activities in which people do things with language. [...] Reading a novel is one of the many instances of language use, one which obviously involves written forms in a fictional setting". 
Resulta igualmente conveniente recordar la validez del uso de textos literarios para la recopilación y el análisis de datos lingüísticos. Así, Jucker (2015: 63) señala que en los últimos años se ha consolidado la idea de que el lenguaje literario puede legítimamente ser objeto de estudio pragmático y aportar interesantes datos que son válidos en sí mismos, sin pretender que sustituyan a los que se obtengan en la comunicación oral espontánea. Muchos otros autores (Lakof y Tannen, 1984; Fowler, 1988; Toolan, 1992; Fludernik, 1993, 1996; Jaworski, 2000; Chapman, 2011, entre otros) han ratificado la utilidad del análisis de datos lingüísticos tomados de textos literarios para la teorización de aspectos de la comunicación en general, pues esos datos nos permiten inspeccionar la competencia pragmática y sociolingüística de los hablantes (en este caso escritores y personajes) y su conocimiento abstracto de lo que se espera de ellos, como usuarios de la lengua, en sus discursos. Como señala Reyes (2002: 58), la literatura no solo nos ofrece representaciones de la lengua, sino que, "por su índole, consiste en la representación de lenguaje en uso". En esta misma línea, Gardner-Chloros y Weston (2015) defienden que, al igual que otras manifestaciones literarias del multilingüismo, el cambio de código literario puede complementar los estudios sobre este fenómeno sociolingüístico, aportando datos sobre la variedad de comportamientos lingüísticos permitidos en una comunidad de habla, sobre el grado de concienciación del autor acerca de esas distintas modalidades y el papel que estas desempeñan en el repertorio lingüístico de la comunidad de hablantes.

En cuanto a la posible consolidación de las voces foráneas empleadas en textos literarios, recordemos que Silva-Corvalán (1989: 174) señaló que es difícil predecir el destino de los préstamos e interferencias de otras lenguas. Ello se debe a que son muchos los factores intra-y extralingüísticos que intervienen en el proceso de difusión de las innovaciones léxicas hasta su inclusión en los registros lexicográficos de la lengua receptora. Por su parte, Hoffer (2002: 18) explica que, cuando una palabra extranjera entra en una lengua, puede permanecer en uso durante siglos, o bien solo durante un tiempo y luego caer en desuso. Normalmente suelen pasar 25 años de uso activo antes de que esa palabra extranjera sea incluida en un diccionario prestigioso, pasando así a ser considerada un préstamo, algo que, como veremos, ya ha ocurrido con algunos hispanismos que aparecen en las novelas. Además, el papel de los textos literarios es reconocido en la introducción del Shorter Oxford English Dictionary on Historical Principles (2007), cuyo objetivo es exponer "the main meanings and semantic developments of words current at any time between 1700 and the present day: those which have been in regular literary or colloquial use at some point in their history; slang or dialect words which are nevertheless likely to be generally encountered through accessible literature or the modern mass media" (énfasis nuestro).

\section{Sobre los préstamos: breves reflexiones}

Además de fascinante, el estudio del préstamo léxico, definido como "palabra que una lengua o dialecto toma de otra u otro" (Trudgill y Hernández-Campoy, 2007: 253), es bastante complejo y heterogéneo. Una dicotomía básica que surge en estos estudios es la que se establece entre 
préstamo y extranjerismo. Según Gómez-Capuz (1998, 2005), en la clasificación general de los préstamos se emplea un criterio clave que tiene que ver con el proceso de adaptación en sí del elemento foráneo, de manera que se contemplan distintas etapas que consideran el grado de integración. Así, se habla de préstamo crudo, préstamo parcialmente integrado y préstamo totalmente integrado. Pero, aunque este suela definirse como el producto final (Anderson, 2004: 17; Kamwangamalu, 1999: 259), Winford (2010: 182) destaca la conveniencia de entender el concepto de préstamo como un proceso, más que un resultado. Esto ayuda mucho cuando se pretende distinguir, por ejemplo, entre casos de préstamo léxico y de cambio de código, algo que resulta muy complicado (Lipski, 2005) y que ha provocado entre los sociolingüistas un intenso debate, aún sin resolver (Gardner-Chloros y Weston, 2015: 196). Son dos las posturas contrarias que se han adoptado en la concepción de los dos fenómenos, encabezadas respectivamente por Poplack y Myers-Scotton. Simplificando la cuestión, puede decirse que Poplack (1980, 1988) y sus asociados (Poplack y otros, 1989) consideran que el préstamo y el cambio de código son procesos muy diferentes, mientras que Myers-Scotton (1988, 1990, 2006) con otros autores (Myers-Scotton y Jake, 2009) defienden la idea de que forman un continuo. Para diferenciarlos, se han empleado criterios tales como la frecuencia de uso entre hablantes monolingües, o el grado de integración morfológica. Así, Kamwangamalu (2000: 60) señala que el préstamo se caracteriza por estar integrado en todos o en cualquiera de los tres niveles: el fonológico, el morfológico y el sintáctico.

Por otra parte, si una determinada forma lingüística de una $L_{1}$ está más integrada en una $L_{2}$ aparecerá en ella con mayor frecuencia. Según esta idea, las palabras que se utilizan más frecuentemente serán préstamos y estarán más disponibles, mientras que las que aparecen con menos frecuencia se clasifican como cambio de código y requieren un cierto grado de competencia bilingüe que no es necesario para el préstamo (Myers-Scotton, 1990, 2006). Por otra parte, Myers-Scotton (1988: 160) recurre al criterio del grado de significado social para distinguir entre un caso de préstamo y otro de cambio de código, mientras que Poplack ${ }^{3}$ (1988: 220) propone que el uso de una palabra o elemento de una lengua extranjera será siempre un caso de cambio de código hasta que empiece a ser utilizado por un número suficiente de hablantes y llegue a ser aceptado por los hablantes nativos y a aparecer en sus diccionarios. Solo entonces podrá considerarse como préstamo, una idea que concuerda con otro acertado criterio que diferencia claramente al préstamo (borrowing) del cambio de código (codeswitching) y que apunta Kamwangamalu (1999: 260), cuando escribe:

Borrowing, whether nonce or integrated does not require or presuppose any degree of competence in two languages; but CS [codeswitching] does. Put differently, borrowing can occur in the

3 Poplack (1980) introdujo el concepto de nonce borrowing, definiéndolo como un elemento lingüístico de otra lengua que un hablante utiliza en su discurso sin adaptarlo al sistema lingüístico de su idioma. 
speech of both monolingual and bilingual speakers alike; whereas CS is strictly speaking a characteristic feature of the linguistic behavior of bilingual speakers. Also, in terms of functions, generally speakers use borrowing to fill lexical gaps in their languages. However, they engage in CS for a variety of reasons, such as expressing in-group solidarity, emphasis, confidentiality, or drawing attention to what is being said.

Con frecuencia, las dificultades para distinguir entre casos de préstamo y casos de cambio de código tienen que ver con que los préstamos pueden mantener el estatus de extranjerismo, o bien con que el cambio de código a menudo se parece a los préstamos por su brevedad (a veces pueden ser palabras sueltas, o expresiones cortas). Por ello algunos investigadores han añadido nuevos términos como por ejemplo "mezcla de códigos" (code-mixing), para cubrir casos intermedios, como el denominado phrasal code-switching, que afecta a la inserción de sintagmas. Sobre la problemática terminológica que rodea al concepto de préstamo y la distinción entre este y el cambio de código, Heath (2001: 432-433) escribe:

...a borrowing is (ideally) a historically transferred form, usually a word (or lexical stem), that has settled comfortably into the target language, while code-switching is (ideally) a spontaneous, clearly bounded switch from sentences of one language to sentences of another, affecting all levels of linguistic structure simultaneously. However, if actual speech patterns in bilingual environments are observed, one finds that borrowing and code-switching are not always so clearly distinct.

Este mismo autor recuerda que el estudio de los préstamos no puede hacerse únicamente desde la perspectiva puramente lingüística, pues los préstamos siempre reflejan el contexto social y cultural en el que se produce la situación de contacto de lenguas (Heath, 2001: 433). Y es el contexto sociocultural lo que en cada caso concreto parece determinar la dinámica que seguirá cada situación de contacto de lenguas; de ahí la importancia que Weinreich (1969) otorgó desde un principio a su estudio. Lo interesante ahora sería cómo conseguir que, en el futuro, a partir de la base que proporcionan los principios descriptivos con los que contamos, se puedan hacer predicciones fiables y generales para el estudio del cambio lingüístico, dado que el contacto de lenguas es ampliamente reconocido como uno de los factores sociolingüísticos que lo produce (Stojicic, 2004: 34).

Otra cuestión relevante es la accesibilidad al préstamo, es decir, la mayor o menor facilidad con que determinadas palabras de una lengua son adoptadas por otra. Así, se sabe que, al tener mayor relación con lo cultural, son las palabras de contenido y no las funcionales las que se prestan más fácilmente (Appel y Muysken, 1987: 171). Según Van Hout y Muysken (1994: 40), los factores que parecen determinar ese grado de "borrowability" son dos: por un lado, la distribución de las categorías gramaticales de las unidades léxicas, tanto en la lengua donante como en la receptora, y, por otro, la acción de una serie de factores operativos que limitan esa accesibilidad al préstamo. Y un problema con el que se enfrenta cualquier intento de diseñar una tipología de los préstamos es el de distinguir entre aquellas palabras de otra lengua que se toman de manera accidental en el discurso (las denominadas interferencias léxicas o non- 
ce borrowings) y aquellas que se integran totalmente en la lengua receptora. Obviamente, como explica Haspelmath (2009: 41), todo préstamo comienza siendo una innovación en el habla de un individuo y el proceso de propagación del vocablo novedoso dentro de la comunidad lingüística es gradual. Posiblemente, en ese proceso se darán casos intermedios entre lo que son préstamos propiamente dichos y los denominados "single-word code-switches" o alternancia de palabras. Estos fenómenos tendrían que denominarse préstamos incipientes o "regular switches" pero nunca "nonce borrowings". En cualquier caso, como bien señala este autor (Haspelmath, 2009: 35), no es fácil explicar los préstamos ni calcular la probabilidad de que determinadas palabras se tomen como préstamos, y la dificultad surge tanto de factores sociales (prestigio de la lengua donante) y actitudinales (actitudes puristas) como gramaticales (es más frecuente el préstamo de sustantivos que de verbos, que requieren una mayor adaptación gramatical).

En este sentido, Van Hout y Muysken (1994: 401) recuerdan la jerarquía de accesibilidad al préstamo que había propuesto el propio Muysken en 1981, jerarquía que se confirma en nuestra recopilación de hispanismos. Es la siguiente:

Nouns - adjectives - verbs - prepositions - coordinating conjuctions - quantifiers - determiners - free pronouns - clitic pronouns - subordinating conjunctions.

\section{Descripción del corpus y metodología}

Los textos analizados en el marco del Proyecto FFI2014-53962-P siguen fielmente los patrones propios del género de la novela rosa y se caracterizan por incluir en sus argumentos un viaje y una estancia más o menos larga en una o varias de las islas atlánticas, sobre todo en Canarias, conocido archipiélago español. Por ello pueden abordarse desde las distintas perspectivas que se sugieren en el título del Proyecto (Discursos, género e identidad...). De hecho, son cuatro las líneas de investigación que contempla este proyecto interdisciplinar: el discurso del paraíso, el discurso de la otredad y la identidad, el discurso de género y el discurso del contacto lingüístico mediante el uso de hispanismos4. Estas líneas surgen de las situaciones de convivencia de lenguas y culturas que presentan las obras pues, con frecuencia, sus protagonistas forman parejas mixtas (héroe canario/español y heroína anglohablante), lo que necesariamente implica un cierto grado de bilingüismo en los textos. En este artículo, enmarcado en la última línea mencionada, analizamos 30 obras publicadas entre 1955 y 2004, ampliando la muestra de novelas con respecto a trabajos anteriores.

4 En menor medida, algunas novelas incluyen también arabismos, italianismos, portuguesismos, así como palabras y expresiones tomadas del francés y el holandés. 
En cuanto a la metodología hay que señalar que esta investigación no ha estado exenta de problemas. De entrada, la localización y adquisición de las novelas no ha sido fácil, pues solo están disponibles en venta online como ejemplares usados y algunas están descatalogadas o no es posible su envío fuera de los Estados Unidos. Por otro lado, en la primera fase de recopilación de textos, comenzamos a digitalizar algunas de las novelas, con el fin de poder analizarlos mediante la aplicación del programa informático Antcon. No obstante, el análisis resultó un tanto problemático5, por lo que finalmente optamos por continuar con la metodología tradicional de lectura minuciosa de las novelas y recopilación manual de los datos para su posterior transcripción digital. Este proceso continúa en marcha en la actualidad con el análisis de los textos que se van localizando y añadiendo al corpus. La elaboración del glosario será posible mediante la creación de una base de datos, actualmente aún en construcción. Inspirándonos en la propuesta de Curell y otros (2009), simplificada en parte, estamos usando un modelo de ficha en la que para cada entrada del glosario se digitalizan datos relativos a los siguientes campos:

1. LEMA: Forma habitual del hispanismo que figura en los diccionarios.

2. ocURREnCia: Forma o formas en las que aparece en los textos.

3. CATEGORÍA GRAMATICAL:

4. DEFINICIÓn: Si cuenta con más de una acepción, estas se numeran y se separan con doble barra vertical.

5. etımología: Se señala la fecha de documentación del hispanismo, indicándose también si existe algún étimo remoto.

6. contexto de uso: Aquí se reproduce el co-texto, es decir, el entorno lingüístico en el que se utiliza el hispanismo.

7. EQUIVALENTE EN INGLÉs: Si lo hubiera.

8. Ámbito temátıco: Se ha diseñado un listado de diversos campos semánticos (cf. González Cruz, 2018).

9. maRCA tipográfica: Se indicará si el hispanismo se escribe en cursiva, entrecomillado, con mayúscula inicial o sin ninguna marca.

10. DISCURSO METALINGÜISTICO: Especificaremos la manera en que el hispanismo se inserta en el discurso, si le sigue una traducción, definición o paráfrasis explicativa, o algún comentario relativo a su carácter de palabra extranjera, si forma parte de un listado, etc.

5 Este programa detectaba como hispanismos palabras como taller, renta, o vale, que resultaron ser la forma comparativa del adjetivo tall (taller), la primera parte de la expresión rent-a-car, y el segundo elemento del nombre del barrio residencial de Londres Maida Vale, respectivamente. Por otra parte, era imposible que el programa discriminara antropónimos y topónimos o que identificara hispanismos si no estaban correctamente escritos, como el adverbio mañana, que a menudo aparecía como manana o bien mahana, y otras formas imprevisibles como merietta, por merienda. 
11. obSERVACIONES: Cualquier otro comentario de interés.

12. AUTOR:

13. TÍTULO DE LA NOVELA:

14. EDITORIAL:

15. AÑO DE PUBLICACIÓN:

16. PÁGINA:

El análisis de toda esta información nos permitirá profundizar, entre otros muchos aspectos, en cuestiones de interés tales como cuáles son las categorías gramaticales y los ámbitos temáticos más proclives al préstamo, qué porcentaje de los hispanismos utilizados no están registrados aún en las fuentes lexicográficas de la lengua inglesa, cuáles son innecesarios y podrían haberse sustituido por equivalentes en inglés (aunque cumplen funciones sociopragmáticas) o bien si las marcas tipográficas reflejan o no el grado de integración del hispanismo.

\section{Resultados preliminares}

Siguiendo el DRAE, que define el hispanismo como "el empleo de vocablos o giros españoles en distinto idioma", hemos detectado un total de 358 hispanismos diferentes en las 30 novelas examinadas. En su mayoría son palabras simples, si bien hay también diversas locuciones y expresiones rutinarias, por lo que conviene hablar de unidades léxicas. A ellas hay que añadir las 29 frases que ofrecemos en la sección 5.5. Por otro lado, los casos de topónimos, odónimos, antropónimos e hipocorísticos son innumerables, por lo que los hemos dejado al margen de la presente compilación para estudiarlos en un futuro trabajo. Nuestro interés principal aquí es comprobar cuáles de esas 358 voces y expresiones hispanas utilizadas en las novelas están registradas en el Oxford English Dictionary online (OED). En función de ello, proponemos cinco categorías para clasificar todas aquellas palabras y expresiones insertadas en el discurso inglés que cualquier hispanohablante reconocería como propias. Seguidamente, dentro de cada categoría, aportamos una relación alfabética de los hispanismos que conformarán las entradas del futuro glosario. Aunque muchas palabras merecerían comentario, debido a los límites de espacio no nos detendremos demasiado en esa labor.

\subsection{Hispanismos registrados como tales (119)}

abuelo; *adios; adobe; alpaca; amigo; arenisca; autopista; avocado; bacalao; barranco; bodega; bodegon; bolero; bonito; caballero; ${ }^{*}$ cabana; calamares; (la) caldera; calentura; calle; campesino; *canto jondo; caña; caramba!; chica; chili/chilli; conquistador; costa; delicada; don; Don Juan; doña; dueña; enamorado; escudo; estancia; fandango; feria; fiesta; finca; fino; flamenco; flamingo; gamba; gazpacho; guava; guacamole; guanche; hacienda; hasta la vista; hidalgo; hombre; loco; macho; madre; madrileño; majas; *major- 
domo; malpais; manta; mantilla; mañana; marina; mariscos; marquesa; matador; medico; merienda (*merietta); meson; mirador; mojo (*mojes); mosquito; muchacha; nada; novio; padre; paella; palomino; papaya; parador; patio; *pension; *peon; peseta; picador; pico; playa; plaza; poncho; privado; pronto; querida; quinta; rancho; *rapido; reja; rioja; sala; salsa; salud!; *sangria; sardana; señor; señora; señorita; señorito; serrano; sierra; siesta; silbo; *simpatico; sombrero; suerte!; tango; tascas; tapas; *Tia Maria; tinto; torero; tornado; tortilla; tristeza; *turron; vino.

Como vemos, los sustantivos suelen figurar en su forma singular en el $O E D$, aunque hay algunas excepciones (calamares, majas, mariscos, tapas, tascas), si bien en las novelas muchos de los lemas aparecen también en plural (conquistadores, ferias, fiestas, mesones). Sorprende el registro de los femeninos marquesa y querida, pero no de marqués y querido, que también se utilizan en los textos. Algunas voces son canarismos (barranco, guanche, malpaís, manta, mantilla) y otras (avocado, chilli/chili, guacamole) proceden del español de Sudamérica, que a su vez las había tomado de leguas indígenas. Casos interesantes son los de avocado y flamingo, que tras sufrir alteraciones, derivaron en otras formas y significados en español. Las formas guayabo y guava alternan en los textos para referirse a la conocida fruta tropical, aunque solo la última está registrada en el OED. Curiosamente, el hispanismo pico se consolidó en inglés a finales del siglo XVI para hacer referencia al pico Teide en la isla de Tenerife (conocido como the Peak), posiblemente por la gran popularidad que tuvo la literatura de viajes anglocanaria (cf. González Cruz, 2013).

Finalmente, haremos unas observaciones semánticas: el OED registra el hispanismo padre (compartiendo origen con el italiano y el portugués) en su acepción de 'sacerdote cristiano', aunque en su etimología se menciona también su sentido de father o 'progenitor masculino'. En las novelas se usa con ambos significados. Hay que decir además que el plural madres es un canarismo que significa ‘semillas' (Corrales y Corbella, 2009) y lo incluimos abajo en la sección 5.4 . EI OED solo registra la forma singular con el significado de mother ('progenitora femenina'), si bien en las novelas aparecen ambas formas (madre y madres) con sus respectivos significados.

\subsection{Voces en uso en español registradas con otro origen (64)}

De los numerosos cognados existentes, incluimos aquí palabras utilizadas en las novelas que coinciden semántica y formalmente ${ }^{6}$ con palabras españolas, aunque tengan otro origen etimológico en inglés, al haberse incorporado a este idioma mediante otras lenguas:

6 Algunas no coinciden de manera exacta ortográficamente (candelabra, chimera, dilemma, idiot, inmoral, inferno, taberna, y otras que carecen de tilde) pero son totalmente reconocibles para el lector hispano. Un caso curioso es el de pareo, lema que el DRAE incluye como préstamo del inglés pareu, originario de Tahití, lo que también indica el OED. En la novela se usa la forma española, pareo, en vez de la inglesa pareu. 
actor; admirable; agenda; area; aroma; aura; café; candelabra; casino; chimera; crater; dilema; director; divan; doctor; emblema; enigma; fado; fatal; (al) fresco; formal; gala; gusto; halo; idiot; inmoral; inferno; jacaranda; jipijapa; laurel; lava; lido; mango; marital; medico; mimosa7; normal; nostalgia; panorama; papa; pareo; pensión; pergolas; personal; pique; portico; protector; rápido; regular; salón; samba; sentimental; sociable; suave; sublime; tanga; taberna; tension; tornado; total; trio; vértigo; villa; vision; vista; vital; vulnerable.

\subsection{Homónimos registrados (5)}

amado, ay, baul, bien, prima.

Como casos especiales, estas palabras muestran una mera coincidencia formal con voces españolas, con las que nada tienen que ver en cuanto a significado y origen. Así, amado (del japonés) significa 'postigo, contraventana', y ay (también escrito aye) solo se usa en los dialectos del norte y en Escocia y significa 'por siempre'. La forma baul se asocia en el OED al sustantivo ball ('baile’) y al verbo bawl ('gritar'), mientras que bien es una variante de bein, adjetivo y adverbio ya obsoleto de origen desconocido usado principalmente en Escocia, que significa 'agradable, cómodo'. Por último, el lema prima figura en el OED como préstamo del latín que hace referencia a la primera página impresa en la que se marca la primera palabra del texto.

\subsection{Hispanismos no registrados (170)}

Aquí enumeramos las unidades léxicas del español usadas en las novelas que no figuran en los registros lexicográficos ingleses. Además de canarismos (madres, tajarastes, tajinastes, timple, zafra), hemos incluido la voz meigas, de origen gallego, muy conocida en España. Aunque, técnicamente, algunas podrían considerarse frases, al consistir en verbos (comprende?; comprendo; entrar), hemos preferido clasificarlas como unidades léxicas al constar solo de una palabra:

adelante; agua; alta costura; amada; amada mía; amor mío; árbitro; ataque cardiaco; avenida-s; avenida maritima; afección; ay, ay!; baúl; bienvenida/o; botánico; buenas noches; buenas noches, amor mío; buenas noches, madrastra; buenas noches, mi corazón; buenas tardes; bueno!; buenos días; buenos días, señora; cabrilla; café con leche; caldo de pescado; canalla; canaria/o; carnaval; carretas; carta blanca; católico; cerdo; cerveza; chica mía; chico; chiquirritica; chiquita; clínico; club náutico; cocido; colérico; comedor; comparsa; comprende?; comprendo; con leche; correosidad; coso; cuellos de almendra; cuñada; de nada; drago; el conquistador; el Teide; (el) diablo; Dios; entrar!; entremeses; favorita; ga-

7 Señala el OED que, aunque etimológicamente surge del latín herba mimosae, este término se formó en inglés mediante acortamiento, tomándose como modelo la lexía española. 
lerías; gimnasio; gofio; gracias; gracias, señora; guapa; guayabo; guerra al cuchillo; hasta mañana; hermana; hola; hombre total; inglesa; isla de los *volcanoes; jameo; jamón serrano; jardín botánico; Juana mía; la calentura; las jacarandas; levante; limonada; lo antes posible; lomo *embushado; madrastra; madres; maldito sea!; maravilloso; mazapanes; medianero; medias; medianoche; (las) meigas; mero; mi cariño; mi corazón; mi mujer; mi niña; mi querida; mi tía; mil pesetas; mío; muchas gracias; *mucho simpático; *mucho tímida; mujer; murgas; muy bien; niebla; nieta/o; nieta mía; niña; observatorio; oh dios!; oh dolor!; papas arrugadas; partida; patio de las muñecas; pequeña; pequeña hermana; pequeña prima; *perdon; perdóneme; pez espada; pico de paloma; policía; por favor; poyos; preciosa; prima mía; pueblo canario; puerto; pulpitos; rancho canario; rastro; refugio; residencia; rondallas; salud, chiquita; salud, mi amigo; salud, querida; señor don; sí and no; sí, señor; sí, señora; sí, señorita; si! si!; sí, *si, señor; *si, *si, señorita; sobrino; supermercado; tajarastes; tajinastes; tediosa; teléfono; tía; tímida; timple; tío; traje; trovador; turrón; un poco; vamos; velo; velocidad moderada; viva España!; zafra.

\subsection{Unidades fraseológicas (29)}

Buenas noches, Cathy. Lo siento mucho!; Cómo está?; Cómo está usted?; Comprende usted?; Desde cuando está allí?; Es tuya la *perdida, no *mia; Está bien; Esta es la señora de quien hablé; Este Benito *Riviero. Mi hijo, *este allí?; Lo prometo; Me alegro; No comprendo, Papá; No es potable; Para la linda señorita pelirroja; Por favor, *vengo conmigo; Quien está a cargo de este?; Sí, comprendo; Sí, Papá, sí. Espero que ella gane; Sí, quiero San Agustín; *Si, usted tiene razón; Te quiero; Te quiero muchísimo; Te quiero mucho; Tengo bastante; Traigame Vd. agua; Usted habla español muy bien!; *Vengo conmigo; Yo comprendo; Yo le sigo.

Como puede observarse, son frecuentes los errores gramaticales (sobre todo de concordancia), que hemos ido marcando con asteriscos. En general, tanto en las frases como en el resto de hispanismos hay variación en el empleo o ausencia de las tildes y los ejemplos aportados aquí los hemos reproducido respetando siempre la forma en que aparecen en el original. Por último, sorprende comprobar que, con demasiada frecuencia, los hispanismos y otros extranjerismos se insertan en las novelas sin traducción, aunque a veces se explica o parafrasea su significado.

\section{Comentario final}

Este artículo ha intentado mostrar el valor de un corpus de novela rosa inglesa ambientado en Canarias como vía para la difusión internacional de hispanismos, dado el gran número de lectores con el que cuenta este género en todo el mundo. En las 30 novelas compiladas para el Proyecto FFl2014-53962-P hemos detectado un total de 358 unidades léxicas totalmente reconocibles para el lector hispano. En este trabajo las hemos clasificado en función de su registro 
en el OED, comprobando que 64 de esas voces se incorporaron al inglés mediante otras lenguas, 170 unidades léxicas no están registradas y 119 (un 33,24\%) constan como hispanismos. Hay también 5 lemas en el OED que resultaron ser solo homónimos de sendos hispanismos, pues nada tienen que ver con las lexías españolas.

Esta destacada presencia de hispanismos en los textos, a los que hay que sumar el empleo de 29 frases, justifica nuestro interés por la creación de un glosario comentado en el que aportaremos datos lexicográficos y mostraremos el uso contextualizado de todas estas voces. Es probable que muchas nunca lleguen a adoptarse como préstamos y mantengan su condición de extranjerismos, al ser usados ocasionalmente por algunos autores como simples casos de cambio de código, buscando el desempeño de ciertas funciones estilísticas o sociopragmáticas (González Cruz, 2018). No obstante, serán necesarios futuros trabajos de rastreo para poder confirmar la consolidación de alguna de las nuevas palabras y expresiones aquí detectadas.

En definitiva, esta investigación ha pretendido contribuir al estudio del español como lengua donante de préstamos, aportando algo de luz al complejo proceso del préstamo. Sin minimizar el papel de otros medios, planteamos la utilidad del registro periódico del uso de hispanismos en textos de la literatura (y la cultura) popular, que concebimos como vías importantes para la difusión internacional de vocabulario español y su posible incorporación a los registros lexicográficos de la lengua inglesa.

\section{Referencias bibliográficas}

\subsection{Fuentes primarias}

AIRLIE, Catherine, 1955: The Valley of Desire, Mills \& Boon.

AIRLIE, Catherine, 1958: Red Lotus, Mills \& Boon.

AIRLIE, Catherine, 1961: Country of the Heart, Harlequin Classic Library.

ARBor, Jane, 1967: Golden Apple Island, Harlequin Books.

Ashe, Jenny, 1987: The Surgeon from San Agustin, Mills \& Boon.

BAIRD, Jaqueline, 2003: At the Spaniards Pleasure, Mills \& Boon.

BrITt, Katrina, 1977: The Villa Faustino, Harlequin Books.

Corrie, Jane, 1980: Island Fiesta, Mills \& Boon.

Danbury, Iris, 1973: The Silver Stallion, Mills \& Boon. 
DANBURY, Iris,1972: Jacaranda Island, Mills \& Boon.

FIELD, Sandra, 2004: Surrender to Marriage, Mills \& Boon.

Howard, Stephanie, 1994: Beware a Lover's Lie, Mills \& Boon.

Kennedy, Nancy, 1979: Secret Longings, Dell Publishing.

Jameson, Claudia, 1987: An Engagement is Announced, Mills \& Boon.

Lane, Pippa, 1978: Nurse in Tenerife, Mills \& Boon.

Mayo, Margaret, 1988: Savage Affair, Harlequin Books.

Maro, Margaret,1990: Mutual Attraction, Mills \& Boon.

Mayo, Margaret,1992: Reluctant Hostage, Mills \& Boon.

Mayo, Margaret,1994: Bitter Memories, Mills \& Boon.

MacLeOd, Jean S., 1977: The Valley of Palms, Mills \& Boon.

MacLeod, Jean S., 1982: Meeting in Madrid, Mills \& Boon.

MACLEOD, Jean S., 1990: Flame of Avila, Mills \& Boon.

NeELs, Betty, 1987: The Doubtful Marriage, Mills \& Boon.

NeEls, Betty,1994: Grasp a Nettle, Mills \& Boon.

Nickson, Hilda, 1987: Voyage of Discovery, Harlequin Books.

Oldfield, Elizabeth, 1986: Beware of Married Men, Mills \& Boon.

PeAke, Lilian, 1983: Night of Possession, Mills \& Boon.

Thorpe, Kay, 1973: An Apple in Eden, Mills \& Boon.

Wentworth, Sally, 1984: Dark Awakening, Mills \& Boon.

Wentworth, Sally,1989: Fire Island, Mills \& Boon.

Corrales, Cristóbal, y Dolores Corbella, 2009: Diccionario Ejemplificado de Canarismos, La Laguna: Instituto de Estudios Canarios. 
Oxford English Dictionary online [http://www.oed.com].

Real Academia Española: Diccionario de la lengua española [http://dle.rae.es].

Shorter Oxford English Dictionary on Historical Principles, 2007.

Speake, Jennifer, y Mark LaFlaur, 2002 online [1999]: The Oxford Essential Dictionary of Foreign Terms in English, Oxford: Oxford University Press [http://www.oxfordreference.com].

\subsection{Fuentes secundarias}

Altchison, Jane, 1991: Language change: progress or decay?, Cambridge: Cambridge University Press.

Algeo, John, 1996: "Spanish loanwords in English by 1900" en Félix Rodriguez Gonzalez (ed.): Spanish Loanwords in the English Language, Berlín / Nueva York: Mouton de Gruyter, 13-40.

Alvar, Manuel, 2000: El español en el sur de Estados Unidos: estudios, encuestas, textos, Madrid: La Goleta / Universidad de Alcalá, D.L.

Anderson, Sheri L., 2004: Status and Solidarity through Codeswitching: Three Plays by Dolores Prida. MA Thesis. Texas A \& M University, College Station [disponible en http://oaktrust.library. tamu.edu/handle/1969.1/536 fecha de consulta: 10 julio de 2016].

ANDERSON, Tyler Kimbal, y Almeida Jacqueline ToRiBı, 2007: "Attitudes towards Lexical Borrowing and intra-sentential codeswitching among Spanish-English Bilinguals", Spanish in Context 4 (2), $217-240$.

Appel, René, y Pieter Muysken, 2005 [1987]: Language Contact and Bilingualism, Londres: Edward Arnold.

ArdiLA, Alfredo, 2005: "Spanglish: An anglicized Spanish dialect", Hispanic Journal of Behavioral Sciences $27(1), 60-81$.

Batista Rodriguez, José J., y Marcos Sarmiento Pérez, 2007: "Hispanismos (y canarismos) en textos decimonónicos alemanes sobre Canarias", Revista de Filología de la Universidad de La Laguna $25,79-88$.

Braunmüller, Kurt, y Juliane House, 2009: Convergence and Divergence in Language Contact Situations, Amsterdam / Filadelfia: John Benjamins Publishing Company.

Cannon, Garland, 1996: "Recent borrowings from Spanish" en Félix Rodriguez Gonzalezz (ed.): Spanish Loanwords in the English Language, Berlín / Nueva York: Mouton de Gruyter, 41-60. 
Chapman, Raymond, 1990: The Language of Thomas Hardy, Hampshire: MacMillan.

Chapman, Siobhan, 2011: Pragmatics, Londres: Palgrave MacMillan.

Cioranescu, Alejandro, 1987: Los hispanismos en el francés clásico, Madrid: Real Academia Española.

ClaRk, Herbert H., 1996: Using Language, Cambridge: Cambridge University Press.

Corbella, Dolores, 1991: "Hispanismos en la obra de Adolphe Coquet: une excursion aux Îles Canaries" en Roberto Dengler Gassin (ed.): Estudios humanísticos en homenaje a Luis Cortés Vázquez, Salamanca: Ediciones Universidad de Salamanca, vol. I, 137-145.

CRYSTAL, David, 2003: The Cambridge Encyclopedia of the English Language, Cambridge: Cambridge University Press.

Curell, Clara, Cristina G. de Uriarte y José M. Oliver, 2009: “Por un glosario de hispanismos en la Literatura de Viajes" en CRistina Solé I CAStells, Montserrat ParRa, Pere Solá y Maria-Carme FigueROLA-CABRol (eds.): Texto y sociedad en las letras francesas y francófonas, Lérida: Universitat de Lleida, 634-641.

Del Valle, José, y Laura Villa, 2006: "Spanish in Brazil: Ianguage policy, business and cultural propaganda", Language Policy 5, 369-92.

Delahunty, Andrew, 2010: Oxford Dictionary of Foreign Words and Phrases, Oxford: Oxford University Press.

Dumitrescu, Dimnita, y Gerardo Pina-Rosales (eds.), 2013: El español en los Estados Unidos: e pluribus unum? Enfoques multidisciplinarios, Nueva York: Academia Norteamericana de la Lengua Española.

Durkin, Phillip, 2014: Borrowed words: A History of Loanwords in English, Oxford: Oxford University Press.

FAIRCLough, Marta, 2003: "El (denominado) Spanglish en Estados Unidos: Polémicas y realidades”, Revista Internacional de Lingüística Iberoamericana I (2), 185-284.

Ferreira Martins, Viviane, 2016: "Las políticas lingüísticas de enseñanza y difusión de español / lengua extranjera (ELE) en el Mercosur”, Onomázein 33, 174-188.

Fludernik, Monika, 1993: The Fictions of Language and the Languages of Fiction. The Linguistic Representation of Speech and Consciousness, Londres / Nueva York: Routledge.

Fludernik, Monika, 1996: "Linguistics and literature: prospects and horizons in the study of prose", Journal of Pragmatics 26, 583-611. 
Fowler, Roger, 1988: "Studying Literature as Language”, Revista Alicantina de Estudios Ingleses $1,81-90$.

Gardner-Chloros, Penelope, y Daniel Weston, 2015: "Mind the gap: What code-switching in literature can tell us about code-switching”, Language and Literature 24 (3), 194-212.

GiRaldo Gallego, Diana Andrea, 2013: "Hispanismos en el Muisca. Bocabulario de la Lengua Chibcha o Mosca. Manuscrito II/2922*", Forma y Función 26 (2), 77-97.

Gómez-Capuz, Juan, 1998: El préstamo lingüístico: conceptos, problemas y métodos, Valencia: Departamento de Filología Española, Universitat de Valéncia, D.L.

Gómez-Capuz, Juan, 2005: La inmigración léxica, Madrid: Arco/libros S.L.

González Cruz, M. ${ }^{a}$ Isabel, y M. ${ }^{a}$ del Pilar González de la Rosa, 2006: “Language and travel: Spanish vocabulary in British travel books", Tesserae. Journal of Iberian and Latin American Studies 12 (2-3), 203-217.

GonzÁlez Cruz, M. ${ }^{a}$ Isabel, 2011a: "Hispanismos y canarismos en los textos de dos viajeras británicas decimonónicas”, Revista de Filología de la Universidad de La Laguna 29, 81-99.

GonzÁlez Cruz, M. a Isabel, 2011b: "Exploring the Canarian contribution to Hispanicisms in English”, Revista Alicantina de Estudios Ingleses 24, 131-152.

González Cruz, M. ${ }^{a}$ Isabel, 2013: "Hispanismos y canarismos en un corpus de textos ingleses sobre Canarias”, LEXIS. Revista de Lingüística y Literatura 37 (2), 229-267.

González Cruz, M. a Isabel, 2015: "Love in paradise: Visions of the Canaries in a corpus of popular romance fiction novels", Oceánide. Journal of the Spanish Society for the Study of Popular Culture SELICUP 7 [disponible en http://oceanide.netne.net/articulos/art7-4.pdf].

GonzÁlez CRUz, M. a Isabel, 2017a: "Exploring the dynamics of English/Spanish codeswitching in a written corpus", Alicante Journal of English Studies 30, Special Issue: English as a Contact Language: Variation and Diffusion, 331-355 [http://dx.doi.org/10.14198/raei.2017.30.12].

GonzÁlez Cruz, M. ${ }^{a}$ Isabel, 2017b: "Conciencia sociolingüística e hispanismos en un corpus de novela rosa inglesa”, SOPRAG 5 (2), 125-149 [https://doi.org/10.1515/soprag-2017-0014].

González Cruz, M. Isabel, 2018: "Hispanismos en el discurso romántico de Harlequin y Mills \& Boon. Ámbitos temáticos y funciones socio-pragmáticas”, Moderna Sprak 1, 157-178.

Gooch, Anthony, 1996: "Aspects of the incidence of hispanicisms in British English: a study in language, history, politics and psychology" en Félix Rodriguez GonzAlez (ed.): Spanish Loanwords in the English Language, Berlín / Nueva York: Mouton de Gruyter, 231-251. 
Grant, Anthony, 2009: "Loanwords in British English" en Martin Haspelmath y Uri Tadmor (eds.): Loanwords in the World's Languages. A Comparative Handbook, Berlín: De Gruyter Mouton, 360-383.

Guijarro Ojeda, Juan Ramón, y Raúl Ruiz Cecilia, 2004: "Attitudes of English People towards Lexical Borrowing", Glosas Didácticas 11, 215-220.

Haspelmath, Martin, 2009: "Lexical borrowing: Concepts and Issues" en Martin Haspelmath y Uri TADMOR (eds.): Loanwords in the World's Languages. A Comparative Handbook, Berlín: De Gruyter Mouton, 35-54.

Heath, Jeffrey, 2001: "Borrowing” en Rajend Mesthrie (ed.): Concise Encyclopedia of Sociolinguistics, Kidlington, Oxford: Elsevier, 432-442.

Hoffer, Bates L., 2002: “Language borrowing and language diffusion: An overview”, Intercultural Communication Studies 11 (4), 1-37.

JaCkson, Howard, y Etienne Zé Amvela, 2004: Words, Meaning and Vocabulary: An Introduction to Modern English Lexicology, Londres: Continuum.

Jaworskı, Adam, 2000: "Silence and Small Talk" en Justine Coupland (ed.): Small Talk, Harlow, Essex: Pearson Education Limited, 110-132.

JUCKER, Andreas H., 2015: "Pragmatics of fiction: literary uses of uh and um", Journal of Pragmatics $86,63-67$.

Kamwangamalu, Nkonko M., 1999: "The state of codeswitching research at the dawn of the new millennium (1): Focus on the global context”, South African Journal of Linguistics 17 (4), 256-277.

Kamwangamalu, Nkonko M., 2000: "The state of codeswitching research at the dawn of the new millennium (2): Focus on Africa", Southern African Linguistics and Applied Language Studies $18(1), 59-71$.

LaCorte, Manel, y Jennifer LeEman (eds.), 2009: Español en Estados Unidos y otros contextos de contacto. Sociolingüística, Ideología y Pedagogía [Spanish in the United States and other contact environments: sociolinguistics, ideology and pedagogy], Madrid / Iberoamericana / Frankfurt: Vervuert.

LAKOF, R. Tolmach, y Deborah TANnen, 1984: "Communicative strategies and metastrategies in a pragmatic theory: the case of Scenes from a Marriage”, Semiotica 17 (3-4), 323-346.

LIMERICK, Philip P., 2017: "Language contact in the US Southeast. The case of Spanish subject expression in an emerging bilingual community in Georgia", Spanish in Context 14 (1), 53-77. 
LIPSKI, John M., 2005: "Codeswitching or Borrowing? No sé so no puedo decir, you know" en Lofti SAYAHI y Maurice WestmoReland (eds.): Selected Proceedings of the Second Workshop on Spanish Sociolinguistics, Somerville: Cascadilla Press, 1-15.

LODARES, Juan R., 1996: "Hispanic tracks in English: cowboys and gold-rushers in the West" en Félix Rodríguez González (ed.): Spanish Loanwords in the English Language, Berlín / Nueva York: Mouton de Gruyter, 157-176.

Lope-Blanch, Juan M., 1990: El español hablado en el suroeste de los Estados Unidos: materiales para su estudio, México: Universidad Nacional Autónoma de México.

Mar-Molinero, Clare, 2000: The Politics of Language in the Spanish-speaking World: from Colonisation to Globalisation, Londres: Routledge.

Mar-Molinero, Clare, 2004: "Spanish as a World Language: Language and Identity in a Global Era”, Spanish in Context 1 (1), 3-20.

Mar-Molinero, Clare, 2013: "The spread of global Spanish: from Cervantes to reggaetón” en Nikolas Coupland (ed.): The Handbook of Language and Globalization, Blackwell Publishing Ltd., 161-182.

Martín, Manuel M., 1994: “Lenguaje y poder: El español en la literatura chicana” en Alberto Moncada-Lorenzo y otros (eds.): El poder hispano, Madrid: Universidad de Alcalá, 487-97.

Morales, Ed, 2002: Living in Spanglish: The Search for Latino Identity in America, Nueva York: St. Martin's Griffin.

Muñoz-Basols, Javier, y Danica Salazar, 2016: "Cross-linguistic lexical influence between English and Spanish", Spanish in Context 13 (1), 80-102.

Myers-SCOtTon, Carol, 1988: "Codeswitching as indexical of social negotiations" en Monica HeLLER (ed.): Codeswitching: Antropological and Sociolinguistic Perspectives, Berlin: Mouton de Gruyter, 151-186.

MyeRs-SCOTton, Carol, 1990: "Codeswitching and borrowing: Interpersonal macrolevel meaning" en Rodolfo Jacobson (ed.): Codeswitching as a Worldwide Phenomenon, Nueva York: Peter Lang, 85-110.

Myers-Scotton, Carol, 2006: Multiple Voices: An Introduction to Bilingualism, Oxford: Blackwell Publishing.

MyeRs-SCOTtOn, Carol, y Janice JAKE, 2009: "A universal model of code-switching and bilingual language processing and production” en Barbara E. Bullock y Almeida Jaqueline ToRiBIo (eds.): The Cambridge Handbook of Linguistic Codeswitching, Cambridge: Cambridge University Press, 336-357. 
Niño-Murcia, Mercedes, y Jason Rothman (ed.), 2008: Bilingualism and Identity. Spanish at the Crossroads with Other Languages, Amsterdam / Filadelfia: John Benjamins Publishing.

Ortiz, Luis A., y Manel LACORTE (eds.), 2005: Contactos y contextos lingüísticos: el español en los Estados Unidos y en contacto con otras lenguas, Madrid: Iberoamericana / Frankfurt: Vervuert.

Parameswaran, Radhika, 1999: "Western Romance Fiction as English-Language Media in PostcoIonial India", Journal of Communication 49 (3), 84-105.

Peñuelas, Marcelino C., 1964: Lo español en el suroeste de los Estados Unidos, Madrid: Cultura Hispánica.

Picone, Michael D., 1996: Anglicisms, Neologisms and Dynamic French, Amsterdam: John Benjamins.

Poplack, Shana, 1980: "Sometimes I'll start a sentence in Spanish Y TERMINO EN ESPAÑOL: Toward a typology of code-switching", Linguistics 18 (7-8), 581-618.

Poplack, Shana, 1988: "Contrasting patterns of codeswitching and transfer" en Monica Heller (ed.): Codeswitching: Antropological and Sociolinguistic Perspectives, Berlín: Mouton de Gruyter, $215-244$

POPLACK, Shana, y otros, 1989: "Distinguishing language contact phenomena. Evidence from Finnish-English bilingualism”, World Englishes 8 (3), 389-406.

Potowskı, Kim, y Jason Rothman (eds.), 2011: Bilingual Youth: Spanish in English-speaking Societies, Amsterdam: John Benjamins.

ReYes, Graciela, 2002: Metapragmática. Lenguaje sobre lenguaje, ficciones, figuras, Valladolid: Universidad de Valladolid.

RocA, Ana, 2000: Research into Spanish in the United States, Somerville: Cascadilla Press.

Rodriguez Gonzalez, Félix (ed.), 1996: Spanish Loanwords in the English Language, Berlín / Nueva York: Mouton de Gruyter.

Rodriguez González, Félix, 1995: "Spanish influence on English word formation. The suffix -ista”, American Speech 70 (4), 421-429.

Rodriguez GonzAlez, Félix, 2001: "Spanish contribution to American English word-stock: An overview", Atlantis: Revista de la Asociación Española de Estudios Anglo-Norteamericanos 23 (2), 83-90. Rodriguez González, Félix (dir.), y Lillo Buades, 1997: Nuevo Diccionario de Anglicismos, Madrid: Gredos. 
Rojas, Vivana, y Juan Piñón, 2014: "Spanish, English or Spanglish? Media strategies and corporate struggles to reach the second and later generation of Latinos", International Journal of Hispanic Media 7, 1-15.

SABIA, Said, y Norddin AchIRI (eds.), 2012: El hispanismo marroquí: balance y perspectivas. Homenaje al profesor Mohamed Khallaf, Fez: Facultad de Letras y Ciencias Humanas Dhar Al Mahraz.

Sánchez-Palencia, Carolina, 1997: El discurso femenino de la novela rosa en lengua inglesa, Cádiz: Publicaciones de la Universidad de Cádiz.

Schultz, Julia, 2018: The Influence of Spanish on the English Language since 1801. A Lexical Investigation, Cambridge: Cambridge Scholar Publishing.

Serjeantson, Mary S., 1935: A History of Foreign Words in English, Londres: Kegan Paul, Trench, Trubner \& Co. Ltd.

SHEARD, J. A., 1954: The Words We Use, Londres: Andre Deutsch.

Silva-Corvalan, Carmen, 1989: Sociolingüística. Teoría y análisis, Madrid: Alhambra Universidad.

Silva-Corvalán, Carmen (ed.), 1995: Spanish in four continents: studies in language contact and bilingualism, Washington, D.C.: Georgetown University Press.

Stavans, Ilan, 2003: Spanglish: The Making of a New Language, Nueva York: HarperCollins.

Stoлıсіс, Violeta, 2004: "Sociolinguistic stimuli to development of the English lexicon: Language contact and social need", Linguistics and Literature 3 (1), 29-36.

Thomason, Sarah, 2010: "Contact explanations in Linguistics" en Raymond Hickey (ed.): The Handbook of Language Contact, Malden, MA: Wiley-Blackwell, 31-47.

ThOMpson, Gregory L., y Edwin M. LAmBor, 2012: Spanish in Bilingual and Multilingual Settings Around the World, Leiden / Boston: Brill.

Toolan, Michael, 1992: "The significations of representing dialect in writing", Language and Literature 1, 29-46.

Trudgill, Peter, y Juan Manuel Hernández-Campoy, 2007: Diccionario de Sociolingüística, Madrid: Gredos.

Turell, M. Teresa, 1986: “La sociolingüística y los estudios ingleses”, Revista Canaria de Estudios Ingleses 12, 35-54. 
Van Hout, Roeland, y Pieter Muysken, 1994: "Modelling lexical borrowability", Language Variation and Change 6, 39-62.

VILAR, María del Mar, 2008: El español, segunda lengua en los Estados Unidos: de su enseñanza como idioma extranjero en Norteamérica al bilingüismo, Murcia: Universidad de Murcia, Servicio de Publicaciones.

Weinreich, Uriel, 1969 [1953]: Languages in Contact. Findings and Problems, The Hague: Mouton.

Winford, Donald, 2010: "Contact and borrowing" en Raymond Hickey (ed.): The Handbook of Language Contact, Malden, Massachusetts: Wiley-Blackwell, 170-187. 